\title{
ENVEJECIMIENTO, GÉNERO Y SEXUALIDAD: APROXIMACIÓN A LOS SIGNIFICADOS SOBRE LA SEXUALIDAD DE MUJERES MAYORES EN LA COMUNA DE VALPARAÍSO
}

\author{
AgING, GENDER AND SEXUALITY: APPROACH TO THE MEANINGS OF \\ SEXUALITY IN OLDER WOMEN IN THE COMUNE OF VALPARAÍSO
}

Fecha recepción: 23 de noviembre 2018 / fecha aceptación: 2 de enero 2019

\section{Ana María Ramos Pinilla ${ }^{1}$,Daniela Yanette Thomson Pulgar ${ }^{2}$ y Nicole Mazzucchelli Olmedo 3}

Cómo citar este artículo:

Ramos, A.M., Thomson, D., y Mazzucchelli, N. (2018). Envejecimiento, género y sexualidad: aproximación a los significados sobre la sexualidad de mujeres mayores en la comuna de Valparaíso. Revista Pensamiento y Acción Interdisciplinaria, 4(2), 8-23.http://doi.org/10.29035/pai.4.2.8

\section{Resumen}

La sexualidad en la vejez es un fenómeno de incipiente desarrollo en el campo gerontológico. Existen creencias y estereotipos que tienden a ubicar a las personas mayores como sujetos asexuados, y a establecer una asociación natural entre envejecimiento y el declinar de la actividad sexual. Sin embargo, la literatura indica que las personas mayores pueden ser activas sexualmente, desarrollando diferentes vivencias en torno a ella. Desde la perspectiva de género se considera que las mujeres han enfrentado mayores dificultades para el goce y la sexualidad en su trayectoria de vida. El artículo expone los resultados de un estudio cualitativo, de tipo descriptivo, cuyo objetivo fue develar los significados atribuidos a la sexualidad de las mujeres mayores participantes de la Oficina del Adulto Mayor, en la comuna de Valparaíso. Participaron seis mujeres mayores, entre sesenta y cuatro y setenta y seis años. Los resultados sugieren que las mujeres mayores significan la sexualidad en un lugar secundario en sus vidas, dando cuenta de experiencias poco positivas, y placenteras, las que se asociaron a un vínculo débil de pareja y a situaciones de violencia que influyen en el valor que hoy le otorgan. A su vez sus experiencias dan cuenta de roles tradicionales de género, y una asociación de la sexualidad con la reproducción y la crianza, aunque reconocen que, al ser mujeres mayores, se han permitido una vivencia de la sexualidad con mayor autonomía, libertad y capacidad de exploración. Se concluye que, la sexualidad se vivencia principalmente desde una dimensión espiritual y afectiva, por sobre la coital. Priorizan las relaciones de cuidado, en su cotidianeidad, y los vínculos afectivos familiares, por sobre las relaciones de pareja. Aún así, valoran un incremento de su capacidad de autonomía, conocimiento de su cuerpo y exploración, la que ha sido desarrollada en su vejez.

Palabras claves: Envejecimiento; Género; Mujeres Mayores; Sexualidad; Significados.

\footnotetext{
${ }^{1}$ Chilena, Trabajadora Social, Licenciada en Trabajo Social, Universidad Viña del Mar. Diplomada en DDHH y no Discriminación UVM. Viña del Mar, Chile. E-mail: anitaramosp.amrp@gmail.com

${ }^{2}$ Chilena, Trabajadora Social, Licenciada en Trabajo Social, Universidad Viña del Mar, Viña del Mar, Chile. E-mail: daniela.thomson@gmail.com

${ }^{3}$ Chilena, Trabajadora Social, Licenciada en Trabajo Social PUCV. Magíster en Gestión Cultural UPLA. Doctoranda en Psicología PUCV. Docente UVM-PUCV. E-mail: nicole.mazzucchelli.o@mail.pucv.cl
} 
Abstract

Sexuality in older age is a phenomenon of incipient development in the gerontological field. There are beliefs and stereotypes that tend to place older people as asexual subjects, and to establish a natural association between aging and declining sexual activity. However, the literature indicates that older people can be sexually active, developing different experiences around it. From the perspective of gender, women are considered to face greater difficulties for enjoyment and sexuality on their life trajectory. The article presents the results of a qualitative descriptive study. The aim of this study was to reveal how significant sexuality is to the elderly female participants of the Office of the Elderly in the commune of Valparaíso. Six women between the age of sixty-four and seventy-six took part in the study. The results suggest that older women put sexuality in a secondary place in their lives, given an amount of experiences that were not very positive and pleasurable, related to a weak bond with their partners and situations of violence that had a great impact on the value that they now give to the subject. At the same time their experiences show evidence of traditional gender roles and an association of sexuality with reproduction and breeding, although they recognize that, being older women, they have allowed themselves to experience sexuality with greater autonomy, freedom and ability of exploration. It is concluded that sexuality is experienced mainly from a spiritual and affective dimension, and not from intercourse itself. They prioritize caring relationships in their daily life and family affective bonds over couple relationships. Even so, they value an increase in their capacity for autonomy, knowledge of their body and exploration, which is more developed now in their old age.

Keywords: Aging; Gender; Meanings; Older women; Sexuality.

\section{Antecedentes Generales}

En la actualidad se estima que existen 962 millones de personas mayores de 60 años a nivel mundial, lo que corresponde al 13\% de la población. Se proyecta que para el año 2050, la cantidad de personas mayores en el mundo superará los 2100 millones (United Nations, 2017) Este aumento, es el resultado de un descenso en las tasas de natalidad y un crecimiento en la esperanza de vida, que se extiende mayormente en las mujeres, caracterizándose el envejecimiento por la feminización de la vejez (Padilla y Apablaza, 2018).

En Chile, los resultados de la última Encuesta Nacional de Calidad de Vida en la Vejez (CASEN, 2017) aplicada a la población mayor de 60 años, da cuenta que ha habido un incremento positivo en la percepción de la calidad de vida de las personas mayores, aunque éste no se declara significativo estadísticamente. A su vez los resultados exponen que el bienestar subjetivo, es el índice que presentó peor evaluación. Este instrumento incorpora por primera vez preguntas asociadas a la sexualidad, en la dimensión de bienestar subjetivo, dando como resultado que un tercio de las personas mayores señaló tener vida sexual activa y el 65\% consideró importante mantenerla en la vejez. 
La calidad de vida de las personas mayores "no se relaciona solamente con factores médicos o biológicos, sino también con otros de índole relacional, dentro de ellos la sexualidad" (Cerquera, Galvis y Cala, 2012, p.74). Pese a ello, es una temática con menor abordaje desde el campo gerontológico, siendo la frecuencia de la actividad sexual, como también los impedimentos orgánicos y funcionales los tópicos más estudiados (Tessler, Schumm, Laumann, Levinson, y O'Muircheartaigh, 2007). Por otra parte, algunos estudios dan cuenta de las percepciones negativas de jóvenes acerca de la sexualidad en la vejez, lo que ha contribuido a considerar a las personas mayores como asexuadas, impotentes y carentes de deseo sexual (Pedrero-García, Moreno-Crespo, y Moreno-Fernández, 2018).

Las investigaciones indican que, en el caso de las mujeres mayores, las desigualdades sociales se acentúan al envejecer, dado que arrastran desventajas y privaciones que han acumulado a lo largo de sus vidas, exponiéndose a situaciones de mayor vulnerabilidad y fragilidad social en relación a los cuidados, el acceso a la salud, los servicios, los bienes económicos entre otros (Aranibar, 2001; Arber y Ginn 1996; Fernández-Mayoralas et al., 2018). Además, las mujeres presentan tasas más altas de discapacidad y mayor presencia de enfermedades incluyendo las de salud mental (Muñoz y Espinosa, 2008).

La sexualidad en las mujeres mayores se encuentra limitada desde un patrón sociocultural de dominación de género, que ha condicionado su desarrollo, autonomía y libertad, por medio de una socialización que las ha ubicado prioritariamente en la esfera privada, de la reproducción, otorgándoles un rol pasivo desde su condición femenina, y donde la experiencia de la sexualidad ha sido limitada por el desconocimiento, y la desinformación con la que han desarrollado la dimensión erótica sexual (Freixas, 2017).

Dado el reducido conocimiento en torno a la experiencia y construcción subjetiva de la sexualidad que realizan las mujeres mayores, el artículo expone los resultados de un estudio desarrollado durante el año 2018, cuyo objetivo fue develar los significados atribuidos a la sexualidad, por parte de las mujeres mayores participantes del taller de piso pélvico, de la Oficina del Adulto Mayor (OCAM), de la llustre Municipalidad de Valparaíso.

\section{Aproximación Teórica - Referencial}

Para el construccionismo social el conocimiento es "construido socialmente", constituido y desarrollado desde operaciones lingüísticas cotidianas, no existiendo una verdad por sobre otra, pues éstas adquieren su validez al alero de una determinada comunidad (López-Silva, 2013). El construccionismo social, estudia los fenómenos a partir de la forma en que las personas experimentan sus vivencias personales, la influencia del contexto social y el conocimiento teórico 
en un determinado tema. Centra su atención en „la relación que existe entre los sujetos que participan de una cultura común, y desde su propia experiencia y subjetividad van construyendo realidades en el lenguaje social" (Donoso, 2004, p 10). Para Gergen, Estrada, y Diazgranados (2007) es necesario superar la mirada dualista cartesiana de sujeto-objeto para avanzar en asumir que el sujeto es activo y constructor de su propia realidad, no existiendo una realidad fuera de él, a la que pudiese acceder.

La posición que asume el sujeto frente a diferentes contextos, la forma en que percibe y vivencia las situaciones y hechos, es un proceso relacionado con las experiencias y significados sociales aprendidos, previamente, en el contexto relacional de intercambios sociales. Es así, como las mujeres mayores van a conceptualizar la sexualidad y su construcción del mundo, a través de sus distintas experiencias, y su participación en ciertas comunidades de significado, donde el conocimiento no solo es construido relacionalmente, sino también se puede definir como un proceso histórico (López-Silva, 2013).

Gergen (1996, en Donoso 2004, p. 11) señala que: "las ideas, los conceptos y los recursos surgen del intercambio social y son mediatizados por el lenguaje", co-produciéndose en un espacio de diálogo compartido. Desde esta perspectiva, en la investigación social es necesario valorar y validar lo que los participantes viven, hacen, interpretan y perciben de sí mismos. Los relatos de las participantes permitirán acceder a las creencias y valores que definen los significados y que van orientando una forma particular de sentir, pensar y actuar cotidianamente (Donoso, 2004). En definitiva, el lenguaje "permite organizar el mundo y dar sentido a la vida, operando como un modo de participación social" ("Como se cita en" Donoso, 2001).

El origen de los significados va a tener sentido en la base misma de las relaciones que se establecen con las personas, desde el momento de nacer y bajo la influencia de las relaciones con su comunidad, "en las acciones coordinadas es que empieza a construir, deconstruir y co-construir de manera constante los significados" (Gergen, 2006, en Arcila, Mendoza, Jaramillo y Cañon, 2010, p. 43-44). Los significados no permanecen inmóviles, y pueden evolucionar y transformarse a través del tiempo, desde la experiencia de los sujetos, pues los sujetos desem-peñan diferentes roles en diversos contextos sociales construyéndose a través de los valores y el conocimiento que se negocia en dicho contexto, pudiendo ser legitimado o no al interior de esa comunidad (López-Silva, 2013).

Por su parte, las experiencias de los sujetos se vinculan a un escenario más idílico, relacionándose con la identidad y con lo que quiero ser desde mi proyecto de vida, donde siempre existiría un "yo incompleto" inacabado desde la comprensión romántica, siendo las expectativas las que se relacionan con ese yo, en búsqueda de lo deseado desde las distintas experiencias con personalidades contenidas (Gergen et al., 2007). 
En el marco de los significados compartidos por una comunidad, el género se refiere a las características otorgadas a las mujeres y hombres definidas por la sociedad, como los roles, normas y las relaciones que existen entre ellos, así como lo esperado de cada uno en dicho rol (OMS, 2018). De esta manera, la edad y el género determinan una forma de clasificación que condiciona una posición en la sociedad, siendo el género una categoría que permite reconocer las situaciones de desigualdad e inferioridad por el hecho de ser mujeres en sociedades patriarcales, y a su vez permite incorporar la carga estigmatizadora que supone ser mujer mayor (De Lemus y Expósito, 2005).

En relación a la sexualidad en las personas mayores, los significados atribuidos evidencian que es una temática cargada de prejuicios y estereotipos cuando es asociada a este grupo (lacub, 2006) indicando que no existiría deseo sexual, que son incapaces de mantener relaciones sexuales, o bien que la sexualidad en la vejez no es positiva ni socialmente valorada. Abundante literatura ha reducido la actividad sexual sólo a la dimensión coital; sin embargo, la actividad sexual también comprende otras actitudes, y otras prácticas. La sexualidad considera elementos que sobrepasan el acto de procrear, adicionando la búsqueda del placer, el deseo, el mundo afectivo y sentimental, esto debido a que las propias personas otorgan un significado a sus experiencias (Vidal y Donoso, 2002, en Molina, 2015)

La sexualidad obedece tanto a una necesidad emocional como fisiológica, que puede influir en el bienestar y calidad de vida de las personas (Árraga, 2016). Para el caso de la gerontología, la sexualidad debe ser abordada desde un discurso moderno que enfrente el mito moralista o puritano que excluye la sexualidad en el envejecimiento, no reduciéndola a la genitalidad y explorando otras dimensiones como el erotismo (lacub, 2006).

En el caso de la sexualidad de las mujeres mayores, ésta ha sido limitada desde un patrón sociocultural restrictivo y castigador, que ha fomentado el desarrollo de una sexualidad al alero de la culpa, el miedo a explorar, gozar, y experimentar activamente, influyendo en el desarrollo de una experiencia sana sexualmente y negando la dimensión inherente a toda persona (Freixas, 2017). 


\section{Metodología}

\section{Diseño}

La investigación tuvo un alcance naturalista e interpretativo, utilizando una metodología cualitativa, en vista que permite comprender al sujeto dentro de su marco de referencia y reconociendo la existencia de múltiples realidades (Silverman, 2016; Taylor y Bodgan, 1994).

El estudio se desarrolló entre los meses de Marzo a Septiembre del año 2018, al interior de la Oficina Comunal del Adulto Mayor. El proceso de inserción al campo implicó la visita y asistencia regular a los talleres de piso Pélvico dictados a contar del Mes de Mayo. Se realizaron notas de campo, en tanto registro detallado y descriptivo (Monistrol, 2018) de las participantes y sus acciones, las que facilitaron la construcción de dimensiones analíticas y la definición de los objetivos específicos en torno a la feminidad, el vínculo afectivo y el erotismo, que se abordan en cada una de las dimensiones descritas posteriormente en el instrumento. A su vez, la inserción al campo permitió el desarrollo de lazos de confianza con las participantes.

La técnica de recolección de información fue la entrevista semi-estructurada o narrativa (Krause, 1995), la que contiene algunos temas predefinidos, pero no presentan una estructura estándar, y es capaz de manejarse de manera flexible, intentando en lo posible acercarse a las situaciones de la vida cotidiana.

El instrumento se compuso por tres dimensiones y catorce preguntas. La primera se relacionó con los valores asociados a la feminidad en el espacio íntimo; la segunda a las experiencias y expectativas otorgadas al vínculo afectivo; y la tercera se asoció a las creencias vinculadas al erotismo las mujeres mayores. Las entrevistas fueron realizadas los meses de Julio y Agosto, y tuvieron una duración promedio de 90 minutos. Fueron realizadas en las viviendas de las participantes, debido a que esto fue solicitado por ellas, en vistas de sentirse más cómodas y en un espacio con mayor privacidad para abordar la temática.

El análisis de información se realizó mediante la propuesta de análisis de contenido de Cáceres (2003), que desarrolla una integración de conceptos para el análisis de contenido categorial, que se sintetizan en torno a seis pasos: la definición de la postura teórica, la organización de la información, la definición de unidades de análisis, la construcción de reglas y códigos y el desarrollo de categorías e integración final de los hallazgos. Para efectos de este artículo, expondremos los resultados centrados en el último paso, es decir categorías e integración de hallazgos. 


\section{Población y muestra}

El colectivo correspondió a mujeres de la tercera edad, participantes del taller de piso pélvico, actividad impartida desde la línea de acción socioeducativa de la Oficina Comunal del Adulto Mayor de la ciudad de Valparaíso.

Se utilizó el muestreo por conveniencia (Flick, 2007), ya que las personas informantes de este estudio, participan en las actividades de OCAM; son personas interesadas en dar cuenta de su experiencia y accedieron voluntariamente a participar del estudio. La muestra estuvo compuesta por 6 mujeres entre los 64 y 76 años. Los criterios de inclusión fueron; mujeres con escolaridad básica completa e incompleta, y con escolaridad media completa e incompleta; mujeres mayores con y sin pareja en la actualidad; mujeres mayores autovalentes y $\sin$ antecedentes de deterioro cognitivo. Se seleccionaron a aquellas mujeres que cumplieran con estos criterios, con el fin de enriquecer los relatos y su grado de heterogeneidad.

\section{Resultados}

A continuación, se presentan los resultados obtenidos del análisis desarrollado centrado en las categorías e integración de los hallazgos (Cáceres, 2003), principalmente en torno a tres dimensiones, que confluyen en una aproximación a los significados otorgados a la sexualidad.

1. El valor asociado a la feminidad en el espacio íntimo de las mujeres mayores se construye a través de la corporalidad y la proyección de su imagen, con la aceptación del cuerpo envejecido.

La proyección de la imagen se encuentra relacionada con un ideal de mujer bella y atractiva que desarrolla ciertas formas de comportamiento, actitudes, etc. (Arango, León, y Viveros, 1995). Es así como la feminidad se encuentra valorada en la medida en que las mujeres sienten que se aproximan a esa construcción canónica, del ser femenina y a ese ideal de belleza construido socialmente.

"serfemenina ponte tú es em... eh... arreglarse el pelo, arreglarse la cara, ponerse una ropa que tú te sientas cómoda y te sientes bien para ti (...) es como una actitud". M2, 67 años.

Se observa como la sexualidad es vista como un "trámite", o una responsabilidad a cumplir, sugiriendo que no se configura como una dimensión constructiva en la pareja. La mujer debe cumplir con el rol marital, y la experiencia del coito para los hombres es representada como la búsqueda de posesión sobre la mujer (Freixas, 2006). 
"mi marido era al lote, era como que tenía que hacer un trámite y nada más". M6, 70 años.

Para el caso de las mujeres el "cuerpo envejecido" se condena, despojándolas de su atractivo social y la valoración de su belleza, la que resulta ser el atributo con el cuál se han permitido ser aceptadas (Sánchez, 2016). Los cambios físicos originados por los embarazos y el avance propio de la edad, provocan variaciones visibles en la constitución del cuerpo, los que no resultan fáciles de aceptar, toda vez que enaltecemos la belleza de la juventud y observamos con desprecio características corporales que dan cuenta de aquellos "signos" de vejez como son, las arrugas, las canas y la imagen del cuerpo envejecido.

"hay cosas que me molestan de mi cuerpo (...) yo creo... que es una inseguridad, porque después de años, yo ya tenía mi guata, mis estrías, mis pechugas ya que no están donde deberían estar". M2, 67 años.

2. Las experiencias y expectativas otorgadas al vínculo afectivo por parte de las mujeres mayores, se relacionan con la comunicación, la sensación de seguridad, y el apoyo emocional.

Los relatos dan cuenta de la importancia de convivir con otras personas, y de la vinculación afectiva con una pareja como una característica asociada a la naturaleza del ser humano. Es así como el amor constituye una de las emociones más intensas en las experiencias de vida de las mujeres mayores, rescatando su importancia.

"el amor a esta edad es bonito, yo no tengo pareja (...) pero es importante en todo ser humano, porque el hecho que te toquen (...) eso es parte de nuestra existencia". M6, 70 años.

Para las participantes, el vínculo afectivo se encuentra relacionado con el apoyo emocional, pudiendo ser ocupado en primera instancia por personas con lazos de consanguinidad o amigos/as, que en su vínculo afectivo de pareja que puedan cumplir el rol de figuras de apoyo (Hansson y Carpenter, 1994).

"(...) con una pareja no, porque siempre he tenido la idea de que algún día van a desaparecer y ahí voy a quedar, en cambio con la familia, aunque él se vaya, siempre va a ser mi nieto. Las parejas en cualquier momento se olvidan de ti y no las ves nunca más". M1, 65 años.

El vínculo afectivo alberga la comprensión mutua, comprensión con el otro y junto al otro, y el compartir emociones, gustos o actividades (Sternberg, 1989), el que no debe ser reducido exclusivamente a la relación de pareja. Los relatos dan cuenta de una experiencia de pareja que no les permite confiar en su permanencia 
y compañía, como si pueden hacerlo con familiares directos, asumiendo además que la pareja es capaz de olvidar y abandonar, por tanto, el vínculo afectivo sería asumido con menor compromiso.

"el ser humano se ha hecho para vivir en pareja y cuando ese espacio no existe, hay que ocuparlo (...) tener amistad, con un hombre o una mujer (...) suplir esa carencia". M6, 70 años.

El apoyo emocional en la pareja, emerge como un elemento esencial, considerando que gran parte de los problemas en las relaciones de pareja, se dan en torno a la comunicación, la incapacidad de escucha, y de desarrollar estrategias de apoyo y confianza con el otro (Hansson y Carpenter, 1994).

"la comunicación en la pareja debiera ocupar el primer lugar... pero no es así. Se espera comprensión, ayuda y contención, más que nada contención, porque cuando tú comunicas, comunicas lo bueno y lo malo". M3, 75 años.

3. Las creencias de las mujeres mayores, asociadas al erotismo se encuentran relacionadas con los prejuicios y percepciones sobre el componente erótico-sexual que ha construido nuestra sociedad, en una tensión con el descubrimiento del disfrute y goce sexual que han vivenciado en su calidad de mujeres mayores.

Se valora la relación de pareja en tanto experiencia de contención y cariño junto al otro, valorando formas de vinculación como el acompañarse y conversar. Las relaciones de pareja cumplen la función de otorgar apoyo emocional, desde un lugar de mayor intimidad y acercamiento emocional, afectivo y comprensivo, desencadenando una nueva mirada sexual en esta etapa de la vida (Sluzki, 1996).

"después las parejas se mantienen por más tiempo y las cosas
se ven de otra forma, uno prefiere estar acurrucado, que te
hagan cariño, que se converse, que te cuenten como les fue".
M5, 64 años.

Se ha descrito que la actividad sexual en la vejez es diferente a etapas previas, debido a la existencia de algunos cambios orgánico-funcionales y a las características propias del envejecimiento (Belsky, 2001). El relato da cuenta que se asume un declive natural de la sexualidad al envejecer, refiriéndose como viejas "calentonas", a aquellas mujeres mayores que sí podrían manifestar deseo sexual.

"yo creo que hay una relación edad-sexualidad y creo que es normal que a uno se le vaya terminando... no sé, por lo menos a mí sí, quizás a otras mujeres no, demás que deben haber viejas calentonas". M5, 64 años. 
Se aprecia un reconocimiento del desgaste natural y la respuesta y apoyo médico al respecto. Sin embargo, las participantes dan cuenta de una aceptación y conformidad respecto a su pareja, existiendo un cambio en las prioridades a raíz de los problemas de salud que limitan o impiden ciertas prácticas sexuales (Gott y Hinchliff, 2003), lo que contribuye en cierta medida a desplazar la dimensión erótica, y la vivencia de la sexualidad, hacia las experiencias previas y sus recuerdos.

"él buscó ayuda (...) el médico le ofreció pastillas y yo le decía que no era necesario (...) le decía que nos quedáramos con los recuerdos (...) yo no exigía". M2, 67 años.

"disfraces (...) pero hay juguetes, aceites... cosas que uno puede ocupar con el marido o pareja... yo hablo de esas cosas con mi nieta, he visto, pero prefiero preguntarle a ella, que quedarme pegada en la vitrina (...) creo que es normal, pero la gente no piensa asi”. M4, 76 años.

Las fantasías sexuales son representaciones imaginarias que estimulan los encuentros sexuales y los acompañan siempre, causando excitación (Mazzel, 2004), de acuerdo a los relatos forman parte de la creatividad en pareja, relacionándose con la estimulación mutua. Sin embargo, se reconocen de cierta forma contenidas, porque se aprecia que el común de las personas no las considera como algo "normal".

"(...) yo me siento sexualmente atractiva... no me siento cómoda con todo mi cuerpo, pero siento que puedo responder a ciertos estímulos y que yo puedo ocasionar ciertos estímulos". M2, 67 años.

Si bien la idea de cuerpo envejecido, y su aceptación emerge nuevamente en los relatos, también se asume la capacidad de movilizar recursos personales y provocar estímulos en el otro, existiendo una aceptación y valoración de su atractivo sexual en la vejez. Es así como la belleza, toma importancia en el espacio íntimo, donde la experiencia del erotismo se establece permitiéndose sentirse mejor y deseable (Arias y lacub, 2015).

Los relatos dan cuenta del reconocimiento de la auto-estimulación como una práctica importante de desarrollar también en la vejez. Si bien la estimulación puede ser muy diferente a otras etapas de la vida, las sensaciones, el deseo de fantasear y seducir, siguen estando vigentes, siendo temas que han sido silenciados a través de los años (Arias y lacub, 2015).

"(...) tanto hombres como mujeres se pueden auto-estimular, a la edad que sea, las ganas siempre están". M6, 70 años. 


\begin{abstract}
"(...) es una necesidad que tienen los hombres y las mujeres también, es normal. Nunca se habla de masturbación femenina, porque la mujer es más retraída, no han tenido la posibilidad, ahora lo hacen abiertamente". M1, 65 años.
\end{abstract}

\title{
Conclusiones
}

Desde los resultados del estudio, se puede concluir que las mujeres mayores significan la sexualidad en un nivel secundario de sus vidas, priorizando los vínculos familiares. Valoran la afectividad, el sentirse queridas y el desarrollo de otras expresiones para afrontar la sexualidad, como son los besos, las caricias y el acompañarse-estar juntos. Los resultados concuerdan con lo propuesto por otras investigaciones (Kalra, Subramanyam, y Pinto, 2018; Can Valle, Sarabia, y Guerrero, 2015; Orte, Vives, y Sánchez, 2015), quienes refieren que la sexualidad en el adulto mayor es una forma de expresar emociones y compromisos, en relaciones en donde se puede compartir a través de la confianza, el amor y el placer, y que esta no se puede reducir simplemente a lo coital.

Pese a esto, se observa que las mujeres mayores siguen posicionándose en un rol tradicional de cuidado, pues pese a que en sus relatos abordan una sexualidad con mayor libertad, en general ésta no se asociaría a sus prácticas. Se aprecia que el rol de cuidado, que suele ser asumido en gran parte por las mujeres mayores (Arroyo, 2010), se centró en la crianza de los hijos y posteriormente se ha centrado en los cuidados de los nietos, lo que favorece que prioricen vínculos afectivos con otros integrantes de la familia, con los que comparten el mayor tiempo, postergando la relación de pareja, o considerándola no prioritaria.

Si bien, la investigación no indagó en la construcción identitaria, Gergen et al. (2007) la entienden en tanto permanencia y transformación. Es así como la experiencia de vida incide o nutre la experiencia actual de las mujeres. En este escenario, emergió como una categoría central y transversal en los relatos, la violencia de género, aunque el estudio no la incluía dentro de sus objetivos de diseño. Sin embargo, emerge como un hallazgo central a propósito de las experiencias previas de violencia, a las que se enfrentaron las participantes. Estás acontecieron específicamente dentro del marco de una relación de pareja, sexo/amorosa, incidiendo tanto en su desarrollo personal-profesional, como en sus proyectos de vida, impactando en las experiencias de sexualidad, las que estuvieron marcadas por contextos de agresión, denostación y miedo, significando en su vejez, la sexualidad desde una experiencia negativa y dolorosa. Esto sugiere el desarrollo de nuevas investigaciones que profundicen en torno a esta trayectoria de violencia y su impacto tanto en el desarrollo de la sexualidad, como en su experiencia de bienestar en la vejez. 
Si bien los resultados exponen que existe una disminución en la función sexual y en el interés que las mismas mujeres mayores declaran en torno a ella, se observa la presencia de algunas prácticas innovadoras y que aportan a su bienestar sexual, como el desarrollar actitudes positivas, el reconocimiento y valoración de sus experiencias previas como aprendizajes que influyen en su autopercepción, y el asumir los cambios propios de la edad, como procesos naturales desarrollando una aceptación de los mismos y de su "yo" en esta nueva etapa. Si bien la capacidad del disfrute se puede ver disminuida por los cambios propios del envejecimiento, las relaciones afectivo-sexuales, se pueden iniciar desde una relación más cariñosa y menos genital (Freixas y Luque, 2009). En este lugar cobra valor la fantasía erótica, pues el estudio permite dar cuenta, que algunas participantes se interesan en explorar en torno a juegos, videos, imágenes, etc. tanto con sus parejas, como con ellas mismas a nivel personal, a través de la práctica de auto estimulación. Estos hallazgos plantean un desafío que nos invita a repensar la construcción de la sexualidad, de cara a las vivencias de las mujeres mayores, avanzando en diseños y propuestas de intervención que amplíen el conocimiento, y la concepción tradicional en su abordaje, y se permitan integrar las propias experiencias en torno a ella.

El conocimiento compartido en torno a la sexualidad, influye directamente en las experiencias y significados con los que las mujeres mayores se enfrentan y relacionan con la sexualidad a lo largo de sus vidas. Las creencias, los valores, las formas de comportamiento sugeridas y que son transmitidas generacionalmente, han reprimiendo a la mujer mayor y han permitido que el hombre desarrolle una sexualidad más libre, sin los sentimientos de culpa, frustración y represión emocional y sexual, como han debido enfrentar las mujeres que hoy son mayores (Freixas, 2017). Algunos autores indican que el erotismo en la vejez, y sobre todo en el caso de las mujeres es un tema sensible "poco abordado desde el punto de vista cultural histórico y político" (lacub, 2006, p.16), favoreciendo que se coarte una expresión libre y autónoma de la sexualidad, más aún cuando se trata de mujeres mayores.

Dentro de las limitaciones del estudio, podemos destacar que la muestra no logró incluir a mujeres mayores de 76 años, principalmente por la falta de adherencia al taller desde el cuál emergió esta investigación, situación que hubiese permitido identificar otros elementos a propósito de su trayectoria generacional. Como segunda limitación podemos agregar el perfil socioeconómico de las participantes, dado que si bien presentaron criterios de inclusión diferenciados para enriquecer la heterogeneidad de la muestra, todas comparten condiciones socioeconómicas similares (beneficiarias de pensión básica solidaria y aporte previsional), sugiriendo entonces que en futuras investigaciones se pueda incorporar un perfil socioeconómico más variado, que pudiera atribuir otros significados a la sexualidad, considerando el carácter situado y material de la experiencia. 
Se puede señalar que la relación entre el envejecimiento y la sexualidad, aún es un tema de bajo interés desde las políticas y programas sociales, existiendo un abordaje que se ha centrado en las dimensiones asociadas principalmente a la salud, no incluyendo una perspectiva integral. A su vez, las estrategias e intervenciones se han caracterizado por asumir objetivos locales, pero sin contar necesariamente con la capacitación y lineamientos para que los equipos amplíen su formación y contribuyan en el desarrollo de la temática.

Finalmente, se aprecia que las mujeres mayores enfrentan la vejez con un conocimiento débil respecto a su capacidad fisiológica y la autopercepción de su propio cuerpo, en una sociedad que aún mantiene rígidos conceptos sobre sexualidad, más aún en el caso de las personas mayores. Uno de los desafíos que debiésemos asumir en el trabajo con personas mayores, es el de poder ir generando mayores herramientas y conocimientos para la apertura en el abordaje de la sexualidad, que permitan considerar el contexto, y que sean capaces de visibilizar las diferentes experiencias que pueden darse en torno a la sexualidad, y cómo estás pueden estar promoviendo o tensionado un mayor bienestar subjetivo en la vejez.

\section{Referencias}

Arango, L., León, M., y Viveros, M. (1995). Género e identidad: ensayos sobre lo femenino y lo masculino. Bogotá, Colombia: Ediciones Uniandes.

Aranibar, P. (2001). Acercamiento conceptual a la situación del adulto mayor en América Latna. Santiago, Chile: CEPAL.

Arber, S., y Ginn, J. (1996). Relación entre género y envejecimiento. Enfoque sociológico. Madrid: NARCEA.

Arcila, P., Mendoza, Y., Jaramillo, J., y Cañon, O. (2010). Comprensión del significado desde Vygotsky, Bruner y Gergen. Revista Diversitas: perspectivas en psicología, 6(1), 37-49.

Arias, C., y lacub, R. (2015). Aspectos positivos en la investigación e intervención con personas mayores. Revista Temática Kairós, 8(20), 1-13.

Árraga, M. (2016). Sexualidad y salud en el adulto mayor. En M. Quintero (Comp.), La salud de los adultos mayores. Una visión compartida (pp. 145-166). Washington: Organización Panamericana de la Salud.

Arroyo, M. (2010). El cuidado en la vejez avanzada. Escenarios y tramas de violencia estructural y de género. Revista de Ciencias Sociales de la Universidad Iberoamericana, 5(10), 1-21. 
Belsky, J. (2001). Psicología del envejecimiento. Madrid: Editorial Paraninfo S.A.

Cáceres, P. (2003). Análisis cualitativo de contenido: una alternativa metodológica alcanzable. Revista de la Escuela de Psicología UC Valparaíso, 2(1), 53-82.

Can Valle, R., Sarabia, B., y Guerrero, J. (2015). Factores psicológicos ysocioculturales en la vida sexual de los mayores. Revista Iberoamericana de las Ciencias de la Salud, 4(8).

CASEN. (2017). Ministerio de Desarrollo Social: Observatorio Social. Recuperado de: $\quad$ http://observatorio.ministeriodesarrollosocial.gob.cl/casenmultidimensional/casen/casen_2017.php

Cerquera, A., Galvis, M., y Cala, M. (2012). Amor, sexualidad e inicio de nuevas relaciones en la vejez: percepción de tres grupos etarios. Revista Psicología: Avances de la disciplina, 6(2), 73-81.

De Lemus, S., y Expósito, F. (2005). Nuevos retos para la psicología social: edadismo y perspectiva de género. Revista Pensamiento Psicológico, 1(4), 33-51.

Donoso, T. (2004). Construccionismo social: Aplicación del grupo de discusión en praxis de equipo reflexivo en la investigación científica. Revista de Piscología de la Universidad de Chile, 13(1), 9-20.

Fernández-Mayoralas, G., Schettini, R., Sánchez-Román, M., Rojo-Pérez, F., Agulló, M., y Joao-Forjaz, M. (2018). El papel del género en el buen envejecer. Una revisión sistemática desde la perspectiva científica. Prisma Social, 21, 150176.

Flick, U. (2007). Introducción a la investigación cualitativa. Madrid: Ediciones Morata.

Freixas, A. (2006). Mujeres y dilemas cotidianos a lo largo del ciclo vital. Revista Crítica, 56(933), 52-55.

Freixas, A., y Luque, B. (2009). El secreto mejor guardado: la sexualidad de las mujeres mayores. Revista Política y Sociedad, 46(1y2), 191-203.

Freixas, A. (2017). Sin reglas. Barcelona: Editorial Capitán Swing.

Gergen, K., Estrada, A., y Diazgranados, S. (Comp.) (2007). Construccionismo social: aportes para el debate y la práctica. Bogotá: Uniandes.

Gott, M., y Hinchliff, S. (2003). How important is sex in later life? The views of older people. Social Science \& Medicine, 56(8), 17-28. 
Hansson, R., y Carpenter, B. (1994). Relationships in old a ge: coping with the challenge of transition. New York: Guilford Press.

lacub, R. (2006). Erótica y vejez: perspectivas de occidente. Buenos Aires: Editorial Paidós.

Kalra, G., Subramanyam, A., y Pinto, C. (2011). Sexuality: Desire, activity and intimacy in the elderly. Indian Journal of Psychiatry, 53(4), 300-306.

Krause, M. (1995). La investigación cualitativa: Un ca mpo de po sibilidades y desafíos. Temas de Educación, 7, 1-19.

López-Silva, P. (2013). Realidades, construcciones y dilemas. Cinta Moebio, 46, 9-25.

Mazzel, J. (2004). Fantasías sexuales en el envejecimiento.IV Congreso Latinoamericano de Geriatría y Gerontología, Simposio: Sexualidad. Santiago, Chile.

Molina, C. (2015). Significados en torno a la sexualidad por parte de un grupo de adultos mayores usuarios de hogares de acogida. Revista de Psicología y Ciencias Afines, 12(2), 22-31.

Monistrol, O. (2018). El trabajo de campo en la investigación cualitativa II. Nure Investigación, 29, 1-4.

Muñoz, F., y Espinosa, J.M. (2008). Envejecimiento activo y desigualdades de género. Atención Primaria, 40(6), 305-309.

OMS. (2018). Género y salud. Recuperado de: http://www.who.int/es/

News-room/fact-sheets/detail/gender.

Orte, M.C., Vives, M., y Sánchez, L. (2015). Amor y sexualidad en la vejez. La historia contada por sus protagonistas. En J. Gazquez, M. Pèrez-Fuentes, M. Montero, I. Mercader y A. Barragán (Eds.), Investigación en salud y envejecimiento (pp.127-133). Andalucía: Editorial Asunivep.

Padilla, C., y Apablaza, M. (2018). Caracterización de la calidad de vida en Chile 1990 y 2015. Centro de políticas públicas - Facultad de Gobierno. Universidad del Desarrollo. Documento №25.

Pedrero-García, E., Moreno-Crespo, P., Moreno-Fernández, O. (2018). Sexualidad en adultos mayores: estereotipos en el alumnado universitario del grado de educación primaria. Formación Universitaria, 11(2), 77-86.

Sánchez, P. (2016). Editorial vejez y género. Algunos conceptos para el análisis y el debate. Research on Ageing and Social Policy, 4(1), 1-21. 
Silverman, D. (Ed.). (2016). Qualitative research. London: Sage.

Sluzki, C. (1996). La red social: Frontera de la práctica sistémica. Barcelona: Editorial Gedisa.

Sternberg, R. (1989). El triángulo del amor. Barcelona: Ediciones Paidós.

Taylor, J., y Bogdan, R. (1994). Introducción a los métodos cualitativos de investigación. Buenos Aires: Editorial Paidós.

Tessler, S., Schumm, P., Laumann, E., Levinson, W., y O'Muircheartaigh, C. (2007). A study of sexuality and health among older adults in the United States. The New England Journal of Medicine, 357, 762-764.

United Nations. (2017). Department of Economic and Social Affairs, Population division: world population prospects; the 2017 revision, key findings and advance tables. Working paper №. ESA/P/WP/248. 11- 16.

Dirección de correspondencia:

Nicole Mazzucchelli Olmedo. Trabajadora Social, PUCV.

Magíster en Gestión Cultural, UPLA. Académica en Universidad de Viña del Mar y Pontificia Universidad Católica de Valparaíso, Chile.

Contacto: nicole.mazzucchelli.o@mail.pucv.cl 